\title{
Potential Role of Novel Myokine in Rats-Induced Metabolic Syndrome
}

\author{
Safinaz E. El-Toukhy ${ }^{1}$, Magdi N.Ashour ${ }^{1}$, Nadia A.Mohamed ${ }^{1}$, Shaimaa A. Gouhar ${ }^{1}$, Noha N. Nazeeh ${ }^{2}$, \\ Eman R. Youness 1, * iD \\ 1 Medical Biochemistry, Departments, Medical Research Division - National Research Centre (ID: 60014618), P.O. 12622, \\ Dokki, Cairo, Egypt \\ 2 Pathology Department, Medical Research Division - National Research Centre (ID: 60014618), P.O. 12622, Dokki, Cairo, \\ Egypt \\ * Correspondence:hoctober2000@yahoo.com (E.R.Y.);
}

Scopus Author ID 55325684200

Received: 5.06.2021; Revised: 15.07.2021; Accepted: 20.07.2021; Published: 8.08.2021

\begin{abstract}
One of the main health problems is metabolic syndrome (MetS). Its incidence elevates with age leading to a higher risk of evolving chronic diseases and cancer. Obesity and insulin resistance was considered the most vital components in its pathogenesis for a long time. This study aims to evaluate serum novel adipokine and myokine to establish the irrelation of insulin resistance and their impact on metabolic syndrome. Four groups of rats were included; the control group (C) fed with a standard diet, the second group $(\mathrm{CI})$ fed on a standard diet and injected daily with irisin $(100 \mathrm{ng} / \mathrm{ml})$ till the end of the experiment. The third group (MetS group) fed on the HCHF diet for 20 weeks and served as a control group. Rats in the fourth group (MetS+I group) were fed on the HCHF diet until they become obese and diabetic, then injected daily with irisin $(100 \mathrm{ng} / \mathrm{ml})$ till the end of the experiment and served as a treated group. Serum levels of obesity and diabetes indices were significantly increased while HDL was significantly decreased in the metabolic syndrome group, but after treatment with irisin, their levels were improved. Both adropin and irisin were significantly decreased, while IL-6 was significantly increased in the same group that was enhanced after irisin treatment. In conclusion, this study demonstrated that lower irisin correlates with the increased risk of increased risk of insulin resistance and MetS. In addition, our results suggested that irisin could have a potential role in glucose metabolism. The relations among increased levels of circulating irisin, insulin resistance, and MetS prevalence may be elucidated with a physiological compensatory contrivance.
\end{abstract}

Keywords: metabolic syndrome; adropin; irisin; interleukin 6.

(C) 2021 by the authors. This article is an open-access article distributed under the terms and conditions of the Creative Commons Attribution (CC BY) license (https://creativecommons.org/licenses/by/4.0/).

\section{Introduction}

Globalization is often accompanied by food habits changes. This contributed to an increase in the incidence of nutrition-associated diseases, including both over- and undernutrition [1].

MetS is a mustering of risk factors for both diabetes mellitus type 2 (T2DM) as well as cardiovascular disease (CVD). Obesity and insulin resistance was considered as the most vital components in its pathogenesis for a long time [2]. Hypertension, atherogenic dyslipidemia, as well as the prothrombotic environment, proinflammatory typically enhanced by obesity, are closely linked with the MetS [3-5].

MetS, as explained by the criteria of National Cholesterol Education Program Adult Treatment Panel III (NCEP-ATP III), is a pleiad of fasting lipoproteins and lipids, blood 
pressure, glucoseand waist circumference abnormalities which were associated with increasing the risk of developing diabetes and CVD [6]. The pervasiveness of MetS ranged between 0.3 \& $26.4 \%$, whereby the increasing number of adolescents and children with MetS partially depended on the used definition. [7].

MetS was one of Western countries' main community health problems; its incidence was augmented by age leading to an advanced risk of developing chronic diseases and cancer $[8,9]$.

In Egypt, the general occurrence of MetS was 16.7\%, which was more frequent among female students [10]. The most effective remediation for MetS is lifestyle modifications, conforming recommendations for a healthy diet with more physical activity expected at weight loss and alleviating the fat/fat-free mass ratio [11]. The Mediterranean diet has been confirmed efficient in patients with MetS [12], cardiovascular diseases [13], diabetes [14] and hypertension [15]. The prevalence of MetS in childhood, which is closely associated with insulin resistance, is a result of abdominal obesity, dyslipidemia, glucose intolerance, and hypertension, increasing worldwide [16]. The congregation of metabolic risk factors starts in childhood [17], and these numerous risk factors resort to keep on from childhood to adulthood [18]. Visceral obesity, cellular dysfunction, and systemic inflammation are risk factors that progressively begin in adolescence and childhood and are related to a high probability of upcoming chronic disease in adulthood.[19].

Adipose tissue is highly documented as a dynamic endocrine organ that regulates insulin resistance and metabolism through adipocytokines production, which could also amend the chronic inflammation related to the MetS [20,21]. It consists of dual obviously altered functional partitions. The prime site of energy storage, white adipose, could also modify muscle and entire-body and/or liver insulin sensitivity through hormonal signals [22]. In newborns, nonshivering thermogenesis in brown adipose tissue (BAT) plays a significant part in preserving body temperature, whereas, in adult persons, this technique appeared to be less declared as BAT was believed to decline with age.[23]. Activated brown adipocytes dawdle energy, causing heat production, i.e., BAT burns fat and raises the metabolic rate, stimulating a negative energy balance. Furthermore, BAT improves metabolic complications as insulin resistance in type 2 diabetes, decreased insulin secretion, and dyslipidemia. [24].

Myokines (i.e., myocyte-secreted proteins) and adipokines (i.e., adipocyte-secreted proteins) have been related to obesity-associated metabolic and vascular diseases. The skeletal muscles communicate with further tissues as the liver, bone, and adipose tissues via hormones secretion, together identified as myokines, whose manner of secretion might be different via the sort and the strength of physical activity [25]. Myokines identification has been directed to suggest that some of the beneficial impacts of exercise on metabolic diseases could be related to myokines and their collaborations with other systems [25].

Irisin is an innovative myokine, first designated by Boström and his colleagues [20]. Firstly identified as a myokine, minor quantities of irisin are also produced and secreted from the adipose tissue or liver [27]. Irisin is a novel myokine that can improve glucose metabolism and reduce obesity as well [20]. The myokines are induced by exercise in humans and rodents, linked with browning in adipose tissue and elevated energy disbursement in mice without prompting food intake and movement, leading to improvement in glucose homeostasis and reducing obesity [17]. Information about the relationship of obesity and glucose metabolic status with irisin in humans and MetS in adults remains debatable [28] [29]. 
Adropin, a peptide hormone, is needed to prevent obesity-associated insulin resistance and for metabolic homeostasis [30]. It is a secretory signal peptide, biologically active when administered to mice and cultured cells. It alters whole-body glucose and lipid metabolism and also stimulates signaling passageways in mammalian cell lines. The function of adropin might also be to preserve regulating endothelial function, the activity of endothelial nitric oxide synthase, the and circulatory system[31]. This study aims to evaluate serum novel adipokine and myokine to establish the irrelation to insulin resistance and their impact on metabolic syndrome.

\section{Materials and Methods}

Animals: Male albino rats from the animal house of the National Research Centre (Cairo, Egypt) 4-6 weeks old, weighing 95 - 106 g, were used. Animal procedures followed the recommendations of the Ethics Committee of the National Research Centre (Cairo, Egypt and the United States National Institutes of Health Guide for Use and Care of Animals in Laboratory (Publication No. 85-23, revised 1985).

\subsection{Diet-induced Metabolic Syndrome in rats.}

Feeding rats with a high-carbohydrate, high-fat (HCHF) diet (western-style diet, Table 1) was used in this study to induce a model that closely mimics the changes observed in human metabolic syndrome (endothelial dysfunction, diabetes, obesity along with nonalcoholic fatty liver disease) according to Loevinger et al., (2007) and A. Hamid et al., (2017) [32,33]. Also, a standard diet was prepared according to A.Hamid et al., (2017) and Reeves (1997) (Table 1) $[34,35]$.

\subsection{Irisin Fc fusion, recombinant.}

Irisin $\mathrm{Fc}$ fusion is a form of irisin that is attached to the $\mathrm{Fc}$ domain of human $\mathrm{IgG}$ in testing the biological activity of irisin. This fusion protein, in principle, renders stability of the protein in vivo and in vitro without significant loss of activity. The lyophilized irisin Fc-fusion, a recombinant protein obtained from PHENIX PHARMACEUTICALS, INC.(GERMANY), will be reconstituted with distilled water to obtain an irisin solution of $100 \mathrm{ng} / \mathrm{ml}$ concentration, which is three times greater than that of normal serum irisin of normal weight rats [20].

Table 1. Component of western-style (high carbohydrate high fat (HCHF) Diet and low-fat diet (standard diet).

\begin{tabular}{l|l|l|l|l} 
Product & \multicolumn{2}{|c|}{ HFF } & \multicolumn{2}{c}{ LF } \\
\hline & gm\% & kcal\% & gm\% & kcal\% \\
\hline Protein & 20 & 17 & 14.2 & 17.7 \\
\hline Carbohydrate & 50 & 43 & 73.1 & 75.9 \\
\hline Fat & 21 & 40 & 4.0 & 9.4 \\
\hline Total ( kcal/gm) & $\mathbf{4 . 6 8}$ & $\mathbf{1 0 0}$ & $\mathbf{3 . 8 5}$ & $\mathbf{1 0 0}$
\end{tabular}

High fat high fructose diet (HFF); Low fat diet (LF) as standard diet.

\subsection{Experimental study design: (Duration of experiment $=20$ weeks $)$.}

The rats were randomly divided into 4 main experimental groups10 rats each. Control group (C) fed with a standard diet, second group (CI) fed on a standard diet and injected daily with irisin (100ng/ ml) till the end of the experiment according to Saleh et al., (2014) [35]. The third group (MetS group) fed on the HCHF diet for 20 weeks and served as a control group. Rats in the fourth group (MetS+I group) were fed on HCHF diet until they become obese and 
diabetic, then injected daily with irisin $(100 \mathrm{ng} / \mathrm{ml})$ till the end of the experiment and served as a treated group.

Sampling: Animals have fasted overnight. Three $\mathrm{ml}$ of blood was aspirated under formalin anesthetized from the peripheral vein of the tail, then 15 min centrifugation at 3000 rpm to get the clear serum stored at $-80^{\circ} \mathrm{C}$ till the evaluation day. Serum lipid profile and glucose level

\subsection{A-Biochemical parameters.}

The glucose level in serum was measured according to the methods of Passing and Bablok (1983) by standard commercial colorimetric enzymatic assays (BioMerieux, Marcy l'Etoile, France; Roche Diagnostics, Basel, Switzerland)[36].

Cholesterol (TC) and triglyceride (TG)levels in serum were measured according to the method of Kwang et al., (2007) and Cole et al., (1984) by standard commercial colorimetric enzymatic assays (BioMerieux, Marcy l'Etoile, France; Roche Diagnostics, Basel, Switzerland)[37,38].

Serum HDL-C was estimated by an enzymatic colorimetric method, according to Movva and Rader (2008) [39]. The kit was supplied from Spectrum Company, Egypt.

\subsubsection{Assessment of insulin.}

Serum insulin level was evaluated using insulin ELISA Kit BioSource, Belgium) according to Yallow and Bauman (1983) [40].

Insulin resistance was calculated from the equation:

Insulin resistance $=$ Fasting glucose $(\mathrm{mg} / \mathrm{dl}) \times$ fasting insulin $/ 22.5(\mu \mathrm{IU} / \mathrm{ml})$ Wallace $e t$ al., (2004) [41].

\subsubsection{Assessment of IL-6.}

The levels of IL-6 (Proinflammatory adipokines)in the samples were measured by using enzyme-linked immunosorbent assay (ELISA) for rats according to Kimura \& Kishimoto (2010) using the manufacturers' protocols (R\&D systems) [42].

\subsubsection{Assessment of irisin.}

The levels of irisin (myokine) in the samples were determined using ELISA kit for rats according to Samy et al. (2015) using the manufacturers' protocols (R\&D systems) [43].

\subsubsection{Assessment of adropin.}

The levels of adropin (myokine) in the samples were determined using Enzyme-linked immunosorbent assay (ELISA) for rats according to Topuz et al. (2013) using the manufacturers' protocols (R\&D systems) [44].

\subsubsection{B-Histopathological examinations:}

Pancreas specimens were dissected immediately after death and fixed in $10 \%$ neutralbuffered formalin saline for at least 72 hours. The specimens were washed with water $30 \mathrm{~min}$ and then dehydrated in ascending grades of alcohol, cleared in xylene, and embedded in paraffin. Serial sections of $6 \mu \mathrm{m}$ thick were cut and stained with Haematoxylin and eosin 
(Drury and Walligton, 1980) for histopathological examination after one month of feeding [45]. Images were captured and processed using Adobe Photoshop version 8.0.

Statistical Analysis: All data were articulated as mean \pm SE. The distribution of the data was verified to be normal using Tests of Normality (SPSS package. Statistical significance was tested by ANOVA followed by Bonferroni post hoc analysis. Pearson's correlation was performed to correlate between the studied parameters. Significance level was considered when $P$ value $<0.001$.

\section{Results and Discussion}

Bodyweight before and after study: Results show no significant difference among study groups regarding weight before the study. The weight gain was highly significantly increased in MetS group with \% of the gain (202\%), while the \% of gain after treatment with irisin was $157.24 \%$ as shown in Table 2. These findings were confirmed by histopathological examinations, as shown in Figure 1.

Table 2. Mean weight before and after treatment of rats in different groups.

\begin{tabular}{c|c|c|c|c} 
& C & CI & MetS & MetS+I \\
\hline Base line weight(gm) & $95.7 \pm 1.22^{\mathbf{a}}$ & $99 \pm 2.2^{\mathbf{a}}$ & $86.1 \pm 4.04^{\mathbf{a}}$ & $90.1 \pm 3.5^{\mathbf{a}}$ \\
\hline Weight gain $($ gm) & $141.3 \pm 7.0^{\mathbf{a}}$ & $132.5 \pm 4.6^{\mathbf{b}}$ & $271 \pm 5.2^{\mathbf{c}}$ & $238 \pm 4.5^{\mathbf{d}}$ \\
\hline \% of weight gain & 44.02 & 33.6 & 202 & 157.24
\end{tabular}

Data presented as mean \pm SE. Significant P-value $<0.001$ and analyzed by one-way analysis of variance dissimilar values (superscripts a, b,c,d) of each column are significantly dissimilar. C: control, CI: control injected irisin, MetS: high-carbohydrate high-fat, MetS+I : high-carbohydrate high-fat injected irisin.

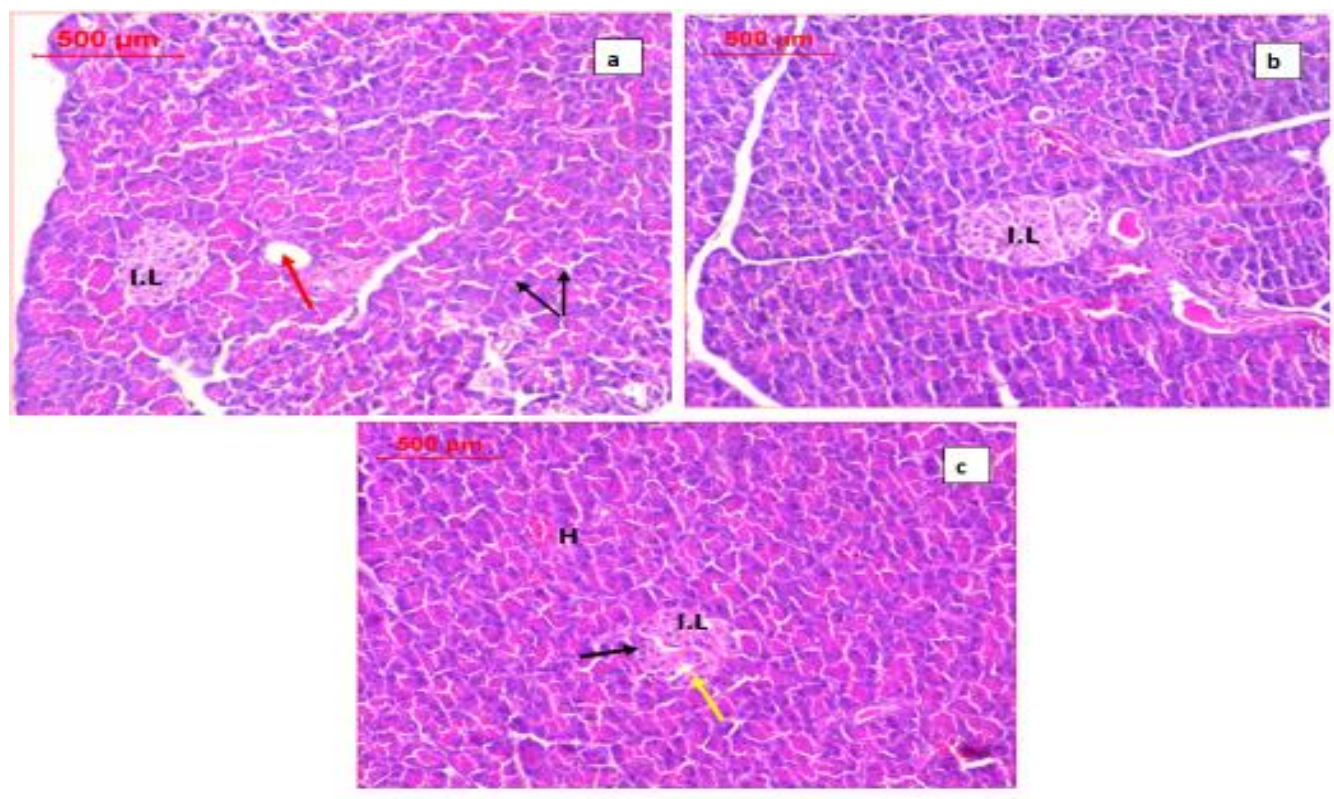

Figure 1. Photomicrography of pancreatic tissue for (a) standard diet group showing the normal architecture formed of the endocrine pancreas in the form of islets of Langerhans (I.L) and exocrine pancreas in the form of acini (black arrows); (b) High fat diet showing the normal architecture formed of the endocrine pancreas in the

form of islets of Langerhans (I.L) with decreased number of islet cells; (c) High fat diet treated with Irisin showing the endocrine pancreas in the form of islets of Langerhans (I.L) with improvement in the roundness of islets and the number of endocrinocytes with minimal widen intercellular spaces (yellow arrows) there are residual endocrinocytes showing signs of degeneration (black arrows) (H\&E200x). 
Serum levels of glucose and lipid profile in different groups after treatment: Serum levels of glucose, cholesterol, TG, insulin, and IR were significantly increased while HDL was significantly decreased in MetSgroup, but after treatment with irisin, the levels of glucose, cholesterol, TG, insulin, and IR were significantly decreased, and the level of HDL was significantly elevated as shown in Table 3.

Table 3. Indices of obesity and diabetes in the studied groups.

\begin{tabular}{c|c|c|c|c} 
& $\mathbf{C}$ & $\mathbf{C I}$ & MetS & MetS+I \\
\hline Glucose (mg/dl) & $82.65 \pm 7.3^{\mathbf{a}}$ & $63.4 \pm 5.2^{\mathbf{b}}$ & $159 \pm 3.9^{\mathbf{c}}$ & $132 \pm 3.1^{\mathbf{d}}$ \\
\hline Cholesterol (mg/dl) & $52 \pm 2.6^{\mathbf{a}}$ & $51.4 \pm 4.1^{\mathbf{a}}$ & $157 \pm 5.2^{\mathbf{b}}$ & $82.2 \pm 3.4^{\mathbf{c}}$ \\
\hline TG (mg/dl) & $52.8 \pm 5.2_{\mathbf{a}}$ & $34.1 \pm 1.3^{\mathbf{b}}$ & $130 \pm 4.1^{\mathbf{c}}$ & $112 \pm 2.3^{\mathbf{d}}$ \\
\hline HDL (mg/dl) & $85.5 \pm 0.7_{\mathbf{a}}$ & $90.2 \pm 2.1_{\mathbf{a}}$ & $54.5 \pm 1.5_{\mathbf{b}}$ & $73.0 \pm 1.2_{\mathbf{c}}$ \\
\hline Insulin (UIu/L) & $0.71 \pm 0.05^{\mathbf{a}}$ & $0.52 \pm 0.5^{\mathrm{b}}$ & $0.99 \pm 0.15^{\mathbf{c}}$ & $0.39 \pm 0.05^{\mathrm{d}}$ \\
\hline Insulin Resistance & $0.152 \pm 0.005^{\mathrm{a}}$ & $0.085 \pm 0.003^{\mathrm{a}}$ & $0.393 \pm 0.025^{\mathrm{b}}$ & $0.131 \pm 0.0092^{\mathrm{c}}$
\end{tabular}

Data presented as mean \pm SE. Significant $\mathrm{P}$-value $<0.001$ and analyzed by one-way analysis of dissimilar variance values (superscripts a, b,c,d) of each column are significantly dissimilar. C: control, CI: control injected irisin, HCHF: high-carbohydrate high-fat, $\mathrm{HCHF}+\mathrm{I}$ : high-carbohydrate high-fat injected irisin.

Effects on serum levels of adropin, IL-6, and irisin: The results summarized in Table 4 showed that both adropin and irisin were significantly decreased while IL-6 was significantly increased in MetSgroup. After treatment with irisin, the levels of adropin and irisin were significantly increased, and the level of IL-6 was significantly decreased.

Table 4. Mean serum levels of adropin (AD), IL-6, and irisin in different groups

\begin{tabular}{c|c|c|c|c} 
& $\mathbf{C}$ & $\mathbf{C I}$ & MetS & MetS+I \\
\hline AD $(\mathbf{p g} / \mathbf{m l})$ & $751 \pm 17.3^{\mathbf{a}}$ & $778 \pm 25^{\mathbf{a}}$ & $623 \pm 24^{\mathbf{c}}$ & $741 \pm 22^{\mathbf{c}}$ \\
\hline $\mathbf{I L - 6}(\mathbf{p g} / \mathbf{m l})$ & $69.0 \pm 1.2^{\mathbf{a}}$ & $62.5 \pm 8.6^{\mathbf{a}}$ & $87.6 \pm 5.9^{\mathbf{b}}$ & $64.1 \pm 4.7^{\mathbf{a}}$ \\
\hline Irisin$(\mathbf{p g} / \mathbf{m l})$ & $19.2 \pm 1.4^{\mathbf{a}}$ & $21.0 \pm 1.5^{\mathbf{a}}$ & $13.1 \pm 1.0^{\mathbf{b}}$ & $25.3 \pm 2.3^{\mathbf{a}}$
\end{tabular}

Data presented as mean \pm SE. Significant P-value $<0.05$ and analyzed by one-way analysis of dissimilar variance values (superscripts a, b,c,d) of each column are significantly different.

Pearson's correlation coefficient showed a statistically significant inverse correlation between irisin and diabetic and obesity indices as well as IL-6. However, it showed a direct correlation with HDL. Moreover, there was a statistically significant direct correlation between diabetic and obesity indices, as shown in Table 5.

Table 5. Correlations in rats feeding with HCHF diet and treated with irisin.

\begin{tabular}{|c|c|c|c|c|c|c|c|c|c|}
\hline & Irisin & IL-6 & Adropin & Insulin & Glucose & IR & HDL & $\begin{array}{c}\text { Cholest } \\
\text { erol }\end{array}$ & TG \\
\hline Irisin & ------- & $-0.640^{*}$ & 0.004 & $-0.917^{* *}$ & $-0.655^{*}$ & $-0.901^{* *}$ & $0.797 * *$ & $-0.933 * *$ & $-0.599 *$ \\
\hline IL-6 & $-0.640^{*}$ & ---------- & -0.189 & 0.539 & $.642^{*}$ & 0.565 & -0.452 & $0.674 *$ & $0.735 * *$ \\
\hline Adropin & 0.004 & 0.189 & ----------- & -0.153 & -0.509 & -0.180 & 0.238 & -0.219 & 0.067 \\
\hline Insulin & $-0.917^{\text {*** }}$ & 0.539 & -0.153 & --------- & $0.686^{*}$ & $0.994^{* * *}$ & $-0.947 * *$ & $0.944 * *$ & $0.715^{*}$ \\
\hline Glucose & $-0.655^{*}$ & $0.642^{*}$ & -0.509 & $0.686^{*}$ & ----------- & $0.780^{* *}$ & $-0.761 * *$ & $0.816^{* *}$ & 0.561 \\
\hline IR & $-0.901^{* *}$ & 0.565 & -0.180 & $0.994^{* *}$ & $0.780^{* * *}$ & -------- & $-0.936 * *$ & $0.955 * *$ & $0.662 *$ \\
\hline HDL & $-.599 *$ & $0.735 * *$ & 0.067 & $0.715^{*}$ & 0.561 & $0.662 *$ & -0.407 & $0.660^{*}$ & -------- \\
\hline Cholesterol & $\overline{0}-933 * *$ & $0.674 *$ & -0.219 & $0.944 * *$ & $0.816^{* *}$ & $0.955 * *$ & $-0.850 * *$ & -------- & $0.660^{*}$ \\
\hline TG & $-0.599 *$ & $0.735 * *$ & 0.067 & $0.715^{*}$ & 0.561 & $0.662 *$ & -------- & $-0.850 * *$ & -0.407 \\
\hline
\end{tabular}

Irisin stimulates the expression of a number of exercise response genes in myotube and pro-myogenic; its injection stimulates hypertrophy significantly because of stimulation of satellite cells and augments protein synthesis, so it is suggested that irisin act as a pro-myogenic 
agent [46, 47]. Privation of upregulation of irisin in insulin resistance status and other components of the metabolic syndrome might hypothetically propose the progress of original cardiac inadequacy in ESRD patients treated with HD [48].

Irisin, a myokine released in response to exercise and a high-fat diet, augments GSIS. Alike to GLP-1, irisin augments promote accrual of $\beta$-cell functional mass and insulin biosynthesis. [49]. It was identified as an innovative hormone that has been proposed to play an important role in energy homeostasis and obesity [25]. Irisin induces mitochondrial brown fat uncoupling protein (UCP1) activity and expression in mice and encourages browning of adipocytes in white adipose tissue depots, thus stimulate elevated total energy expenditure, thermogenesis, and reduced obesity [50]. Overexpression of irisin in animals fed a high-fat diet caused improvement of glucose tolerance and hyperinsulinemia.

In the current study, irisin levels were significantly decreased in the metabolic syndrome group that was diabetic, obese, and overweight compared to those of the control with normal weight as the primary target of irisin is the adipose tissue. Levels of irisin also have a significant inverse association with diabetes as determined by glucose, insulin, and insulin resistance. In line with these findings, Bostrom et al. 2012 revealed that irisin concentrations were significantly decreased in obese/overweight prepubertal children with MetS than those with adipose tissue depots in a well-identified process as white fat browning [20]. Also, it elevates total energy disbursement in rodents and the irisin expression in mice that were fed a high-fat diet leading to a significant improvement in glucose tolerance and a reduction in fasting insulin levels. As well as, irisin encouraged an increase in thermogenesis that is associated with glucose metabolism, body weight, and insulin sensitivity in mice.

The correlation between glucose homeostasis and irisin was suggested to be linked with augmented oxidation of fatty acid and utilization of glucose through the adenosine monophosphate-activated protein kinase (AMPK) signaling pathway in a diabetic mouse model [51,52]. It was reported that diminished irisin levels were related to the increase of insulin resistance and its related diseases, such as MetS and T2DM [53]. Our results indicated that decreases in irisin levels are significantly related to MetS and confirm the preceding studies.

Increased production of endogenous cholesterol is the main factor contributing to hypercholesterolemia in obesity. Plasma concentrations of adropin also seemed to be decreased in cases of obesity-related hypercholesterolemia, a condition in which cholesterol synthesis is augmented. This indicates a relationship of obesity with low plasma adropin levels and elevated metabolic risk factors in humans. In mouse models of obesity, adropin production decreases is a secondary impact of weight gain [20]. As showed in the present work, there was a statistically significant reduction in plasma adropin levels in MetS group that was improved when treated with irisin. Ghoshal et al., 2018 also suggested a relationship between plasma adropin concentrations and obesity [54].

Obesity is associated with lower circulating adropin concentrations in humans. Adropin-deficient mice display impaired glucose tolerance with diet-induced obesity, dyslipidemia, hyperinsulinemia, and insulin resistance [20]. Furthermore, recombinant adropin administration reversed dyslipidemia and insulin resistance in mice [25].

Investigating adropin-deficient rats indicates that the peptide hormone is needed for metabolic homeostasis, specifically preventing dyslipidemia, maintaining insulin sensitivity, and protecting against impaired glucose tolerance. In addition, mounting evidence indicates 
that adropin may act as a hormone in regulating metabolic homeostasis, in part by controlling substrate (glucose and fatty acid) metabolism in skeletal muscle [55].

A significant increase in plasma adropin levels was observed after RYGB (Roux-en-Y gastric bypass), indicating that reverse of the metabolic syndrome is associated with a reversal of low plasma adropin levels and also with obesity [56,57].

Our study demonstrated that lower irisin is related to increased risk of insulin resistance and with a raised probability of having MetS. In addition, our results recommended that irisin might play a potential role in glucose metabolism. The relations between insulin resistance, MetS prevalence, and high levels of circulating irisin may be elucidated with a physiological compensatory mechanism, which could result in elevated irisin levels because of an essential declined sensitivity to irisin's effects (i.e., MetS and obesity being irisin-resistant states, resembling insulin resistance) or possible alternative suggestion as increased production via the elevated fat tissue and muscle in obesity. The potential mechanisms supportive to these associations must be explained by further mechanistic studies.

\section{Conclusions}

Our study demonstrated that lower-level irisin is related to increased risk of insulin resistance and with a raised probability of having MetS. In addition, these results suggested that irisin could play a potential role in glucose metabolism. The relations between insulin resistance, MetS prevalence, and high levels of circulating irisin may be elucidated with a physiological compensatory mechanism, which could result in elevated irisin levels because of an essential declined sensitivity to irisin's effects (i.e., MetS and obesity being irisin-resistant states, resembling insulin resistance) or possible alternative suggestion as increased production via the elevated fat tissue and muscle in obesity. The potential mechanisms supportive to these associations must be explained by further mechanistic studies.

\section{Funding}

National Research Centre has funded project number 11010131.

\section{Acknowledgments}

The authors acknowledge National Research Centre, Egypt, for its assistance

\section{Conflict of interests}

None.

\section{References}

1. Belhayara, M. I.; Zoheir,M.; Hamdaoui, M. S.; Bachaoui,M.; KherouaO.; Willy, J. M.The Metabolic Syndrome: Emerging Novel Insights Regarding the Relationship between the Homeostasis Model Assessment of Insulin Resistance and other Key Predictive Markers in Young Adults of Western Algeria. Nutrients 2020, 12, 727, https://doi.org/10.3390/nu12030727.

2. Reaven, G.M.; Insulin resistance: the link between obesity and cardiovascular disease. Med Clin North Am. 2011, 95, 875-892, https://doi.org/10.1016/j.mcna.2011.06.002.

3. Carr, D.B.; Utzschneider, K.M.; Hull, R.L.; et al. Intra-abdominal fat is a major determinant of the National Cholesterol Education Program Adult Treatment Panel III criteria for the metabolic syndrome. Diabetes 2004, 53, :2087-2094, https://doi.org/10.2337/diabetes.53.8.2087. 
4. Alessia, A.; and Francesco C. Obesity and Diabetes: A Sword of Damocles for Future Generations. Biomedicines 2020, 8(11), 478, https://doi.org/10.3390/biomedicines8110478.

5. Arun, K. Ch.; Metabolic Syndrome or Insulin Resistance: Evolution, Controversies and Association With Cardiovascular Disease Risk. Indian Journal of Clinical Cardiology 2020, 1(2) 77-85, https://doi.org/10.1177/2632463620935030.

6. Grundy, S. Expert Panel on Detection, Evaluation, and Treatment of High Blood Cholesterol in Adults. Executive Summary of the third report of The National Cholesterol Education Program (NCEP). Expert Panel on Detection, Evaluation, and Treatment of High Blood Cholesterol In Adults (Adult Treatment Panel III) JAMA 2001, 285, 2486-97, https://doi.org/10.1001/jama.285.19.2486.

7. Carolin, R.; Benedicta, N.; Nkeh-Chungag, Per Morten F.; Nandu G. The prevalence of pediatric metabolic syndrome - a critical look on the discrepancies between definitions and its clinical importance. International Journal of Obesity 2021, 45,12-24, https://doi.org/10.1038/s41366-020-00713-1.

8. Beltrán-Sánchez, H.; Harhay, M.O.; Harhay, M.M.; McElligott, S. Prevalence and trends of metabolic syndrome in the adult U.S. population, 1999-2010. J. Am. Coll. Cardiol 2013, 62, 697-703, https://doi.org/10.1016/j.jacc.2013.05.064.

9. Fathimah, S. S.; Dicky, Tahapary, L.; Stella, T.; Erliyani, S.; , Frits, R.; De Mutsert, R. The prevalence of metabolic syndrome and its association with body fat distribution in middle-aged individuals from Indonesia and the Netherlands: a cross-sectional analysis of two population-based studies. Diabetology \& Metabolic Syndrome 2020, 12, 2, https://doi.org/10.1186/s13098-019-0503-1.

10. Mahrous, O.; El Shazly H.M.; Badr S.A.; Ibraheem R.A.; Kasemy Z.A.; El Sheikh G.M. Epidemiology of metabolic syndrome in Menoufia University students. Menoufia Medical Journal 2018, 31(3), 839-845, https://www.mmj.eg.net/article.asp?issn=1110-

2098; year=2018; volume=31;issue=3; spage $=839$; epage $=845$; aulast=Mahrous .

11. Yamaoka, K.; Tango, T. Effects of lifestyle modification on metabolic syndrome: A systematic review and meta-analysis. BMC Med 2012, 10, https://doi.org/10.1186/1741-7015-10-138.

12. Kastorini, C.-M.; Milionis, H.J.; Esposito, K.; Giugliano, D.; Goudevenos, J.A.; Panagiotakos, D.B. The effect of Mediterranean diet on metabolic syndrome and its components: A meta-analysis of 50 studies and 534,906 individuals. J. Am. Coll. Cardiol 2011, 57, 1299-1313, https://doi.org/10.1016/j.jacc.2010.09.073.

13. Estruch, R.; Ros, E.; Salas-Salvadó, J.; Covas, M.I.; Corella, D.; Arós, F.; Gómez-Gracia, E.; Ruiz-Gutiérrez, V.; Fiol, M.; Lapetra, J.; et al. Primary prevention of cardiovascular disease with a Mediterranean diet. N.Engl. J. Med 2013, 368, 1279-1290, https://doi.org/10.1056/NEJMoa1200303.

14. Salas-Salvadó, J.; Bulló, M.; Babio, N.; Martínez-González, M.Á.; Ibarrola-Jurado, N.; Basora, J.; Estruch, R.; Covas, M.I.; Corella, D.; Arós, F.; et al. Reduction in the incidence of type 2 diabetes with the Mediterranean diet: Results of the PREDIMED-Reus nutrition intervention randomized trial. Diabetes Care 2011, 34, 14-19, https://doi.org/10.2337/dc10-1288.

15. Estruch, R. Anti-inflammatory effects of the Mediterranean diet: The experience of the PREDIMED study. Proc. Nutr. Soc. 2010, 69, 333-340, https://doi.org/10.1017/S0029665110001539.

16. Oh, K.; Jang, M.J.; Lee, N.Y.; Moon, S.; Lee, C.G.; et al. Prevalence and trends in obesity among Korean children and adolescents in 1997 and 2005. Korean $J$ Pediatr 2013, 51, 950-955, https://doi.org/10.3345/kjp.2008.51.9.950.

17. Chen, W.; Bao, W.; Begum, S.; Elkasabany, A.; Srinivasan, S.R. et al. Age-related patterns of the clustering of cardiovascular risk variables of syndrome $\mathrm{X}$ from childhood to young adulthood in a population made up of black and white subjects: the Bogalusa Heart Study. Diabetes 2000, 49, 1042-1048, https://doi.org/10.2337/diabetes.49.6.1042.

18. Ford, E.S.; Giles, W.H.; Mokdad, A.H. Increasing prevalence of the metabolic syndrome among u.s. Adults. Diabetes Care 2004, 27, 2444-2449, https://doi.org/10.2337/diacare.27.10.2444.

19. Mark, D. DeBoer Assessing and Managing the Metabolic Syndrome in Children and Adolescents.Nutrients. 2019, 11(8): 1788, https://doi.org/10.3390/nu11081788.

20. Bostrom, P.; Wu, J.; Jedrychowski, M.P.; Korde, A.; Ye, L.; Lo, J.C.; Rasbach, K.A.; Bostrom, E.A.; Choi, J.H.; Long, J.Z.; Kajimura, S.; Zingaretti, M.C.; Vind, B.F.; Tu, H.; Cinti, S.; Hojlund, K.; Gygi, S.P.; Spiegelman, B.M. A PGC1-alpha-dependent myokine that drives brown-fat-like development of white fat and thermogenesis. Nature 2012, 481, 463-468, https://doi.org/10.1038/nature10777.

21. Kojta, I.; Chaci 'nska M. and Błachnio-Zabielska, A. Obesity, Bioactive Lipids, and Adipose Tissue Inflammation in Insulin Resistance. Nutrients 2020, 12, 1305, https://doi.org/10.3390/nu12051305. 
22. Cinti, S. Between brown and white: novel aspects of adipocyte differentiation. Ann Med. 2011, 43, 104-115, https://doi.org/10.3109/07853890.2010.535557.

23. Kuryłowicz, A.; Puzianowska-Ku 'znicka, M. Induction of Adipose Tissue Browning as a Strategy to Combat Obesity. Int. J. Mol. Sci. 2020, 21, 6241, https://doi.org/10.3390/ijms21176241.

24. Katarzyna, M.; and Adam, K. Brown Adipose Tissue and Its Role in Insulin and Glucose Homeostasis. Int J Mol Sci. 2021, 22, (4), 1530, https://doi.org/10.3390/ijms22041530.

25. Polyzos, S.A.; Kountouras, J.; Shields, K.; Mantzoros, C.S. Irisin: a renaissance in metabolism? Metabolism 2013, 62, 1037-1044, https://doi.org/10.1016/j.metabol.2013.04.008.

26. Kwon, J. H.; Kyoung, Min M.; and Kyueng-Whan, M. Exercise-Induced Myokines can Explain the Importance of Physical Activity in the Elderly: An Overview. Healthcare 2020, 8, 378, https://doi.org/10.3390/healthcare8040378.

27. Roca-Rivada A.; Castelao C.; Senin, L.L.; Landrove, M.O.; Baltar, J.; Belen, Crujeiras, A.; Seoane, L.M.; Casanueva, F.F.; Pardo, M. FNDC5/irisin is not only a myokine but also an adipokine. PLoS ONE 2013, 8, e60563, https://doi.org/10.1371/journal.pone.0060563.

28. Zhang, R.; Fu, T.; Zhao, X.; Qiu, Y.; Hu, X.; Shi, H.; Yin, X. Association of Circulating Irisin Levels with Adiposity and Glucose Metabolic Profiles in a Middle-Aged Chinese Population: A Cross-Sectional Study. Diabetes, Metabolic Syndrome and Obesity: Targets and Therapy 2020, 13, 4105-4112, https://doi.org/10.2147/DMSO.S275878.

29. Huh, J.Y.; Siopi, A.; Mougios, V.; Park, K.H.; Mantzoros, C.S. Irisin in response to exercise in humans with and without metabolic syndrome. J Clin Endocrinol Metab 2015, 100, 453-457, https://doi.org/10.1210/jc.2014-2416.

30. Butler, A.; Tam, C.; Stanhope, K.; Wolfe, B.; Ali, M.; O'Keeffe, M.; St-Onge, M.; Ravussin, E.; Havel, P. Low Circulating Adropin Concentrations with Obesity and Aging Correlate with Risk Factors for Metabolic Disease and Increase after Gastric Bypass Surgery in Humans . The Journal of Clinical Endocrinology \& Metabolism 2012, 97(10), 1, https://doi.org/10.1210/jc.2012-2194.

31. Chen, S.; Zeng,, K.; Liu, Q.C.; Guo, Z.; Zhang, S.; Chen, X. R.; et al., Adropin deficiency worsens HFDinduced metabolic defects. Cell Death \& Disease 2017, 8(8), e3008, https://doi.org/10.1038/cddis.2017.362.

32. Loevinger, B. L.; Muller, D.; Alonso, C.; Coe, C. L. Metabolic syndrome in women with chronic pain. Metabolism 2007, 56(1), 87-93, https://doi.org/10.1016/j.metabol.2006.09.001.

33. A. Hamid, F. F.; Habib, D. F.; El-Arab, A. M. E.; Mohamed, N. A.; Mohamed, R. A. Effect of Western style diet on nlrp3 inflammasome and its correlation with metabolic syndromes. International Journal of Clinical Chemistry and Laboratory Medicine 2017, 3, 2, 1-8, https://doi.org/10.20431/2455-7153.0302001.

34. Reeves, P.G.; Nielsen, F.H.; Fahey, G.C. AIN-93 purified diets for laboratory rodents: final report of the American Institute of Nutrition ad hoc writing committee on the reformulation of the AIN-76A rodent diet. $J$ Nutr. 1993, 123(11), 1939-1951, https://doi.org/10.1093/jn/123.11.1939.

35. Saleh, O.; Majeed, M.; Oreaby, G. Descriptive consideration of serum irisin levels various factors, obesity, type 2 diabetes mellitus, pre-diabetic status, gender, and athletics. J Diabetes Metab.2014, 5(471), 2, https:/www.longdom.org/open-access/descriptive-consideration-of-serum-irisin-levels-various-factorsobesity-type-diabetes-mellitus-prediabetic-status-gender-and-athletics-2155-6156.1000471.pdf.

36. Passing, H.; and Bablok, W.A. New biochemical procedure for testing the equality of measurements from two different analytical methods: Journal of Clinical Chemistry and Clinical Biochemistry.1983, 21, 709720, https://doi.org/10.1515/cclm.1983.21.11.709.

37. Kwang, O.;Young, A ; Hyesung, L. Isoflavone rich bean sprout cookie improves lipid metabolism in hyperlipidemic rat: The FASEB Journal. 2007, 1(21), 846 - 848, https://doi.org/10.1096/fasebj.21.6.A1087a.

38. Cole, T.G.; Kuisk, I.; Patsch, W.; and Schonfeld, G. Effects of high cholesterol diets on rat plasma lipoproteins and lipoprotein cell interactions: Lipid Res. 1984, 25, 593-603, https://pubmed.ncbi.nlm.nih.gov/6431045/.

39. Movva, R.; Rader, D.J. Laboratory assessment of HDL heterogeneity and function. Clin. Chem. 2008, 54, 788-800, https://doi.org/10.1373/clinchem.2007.101923.

40. Yallow, F.; and Bawman, W.A. Plasma insulin in health and disease. The American Journal of Medicine 1961, 31, 874-881, https://doi.org/10.1016/0002-9343(61)90029-8.

41. Wallace, T. M.; Levy, J. C.; Matthews, D. R. Use and abuse of HOMA modeling. Diabetes Care 2004, 27(6), 1487-1495, https://doi.org/10.2337/diacare.27.6.1487. 
42. Kimura, A.; and Kishimoto, T. IL-6: Regulator of Treg/Th17 balance: European Journal of Immunology 2010, 40, 1830-1835, https://doi.org/10.1002/eji.201040391.

43. Samy, D.M.; Ismail, C.A.; Nassra R.A. Circulating irisin concentrations in rat models of thyroid dysfunction - Effect of Exercise: Metabolism 2015, 64(7), 804-813, https://doi.org/10.1016/j.metabol.2015.01.001.

44. Topuz, M.; Celik, A.; Aslantas ,T.; Demir, A.K.; Aydin, S.; Aydin, S. Plasma adropin levels predict endothelial dysfunction like flow-mediated dilatation in patients with type 2 diabetes mellitus: J Investig Med. 2013, 61(8), 1161-4, https://doi.org/10.2310/JIM.0000000000000003.

45. Drury, R.A.; and Wallington E.A. Coreleton's Histological technique 4th edition. Oxford, Oxford University Press. 1980, 5th ed.,vi, 520 p. : ill, https://www.worldcat.org/title/carletons-histologicaltechnique/oclc/598937579.

46. Reza, M.M.; Subramaniyam, N.; Sim, C.M.; Ge, X.; Sathiakumar, D.; McFarlane, C.; Sharma, M.; Kambadur, R.; Irisin is a pro-myogenic factor that induces skeletal muscle hypertrophy and rescues denervation-induced atrophy. Nat. Commun. 2017, 8, 1104, https://doi.org/10.1038/s41467-017-01131-0.

47. Barbalho, S. M.; Uri, Adrian, P. F.; Ricardo, J. T.; Ricardo, de Alvares G.; Elen, L. G.; Cláudia Rucco P. D.; Buchaim Daniela V.; et al. Physical Exercise and Myokines: Relationships with Sarcopenia and Cardiovascular Complications. Int. J. Mol. Sci. 2020, 21, 3607, https://doi.org/10.3390/ijms21103607.

48. Kałużna, M.; Pawlaczyk, K.; Schwermer, K.; Hoppe, K.; Człapka-Matyasik, M.; Ibrahim A. Sawicka-Gutaj, N.; Minczykowski, A.; and Ziemnicka, K. Adropin and irisin: New biomarkers of cardiac status in patients with end-stage renal disease? A preliminary study. Adv Clin Exp Med. 2019, 28(3), https://doi.org/10.17219/acem/81538.

49. Marrano, N.; Biondi, G.; Borrelli, A.; Cignarelli, A.; Perrini, Sebastio.; Luigi, L.; Giorgino, F.; and Natalicchio, A. Irisin and Incretin Hormones: Similarities, Differences, and Implications in Type 2 Diabetes and Obesity. Biomolecules 2021, 11, 286, https://doi.org/10.3390/biom11020286.

50. Fain, J.N.; Company, J.M.; Booth, F.W.; et al. Exercise training does not increase muscle FNDC5 protein or mRNA expression in pigs. Metabolism. 2013, 62(10), 1503-1511, https://doi.org/10.1016/j.metabol.2013.05.021.

51. Xin, C.; Liu, J.; Zhang, J.; Zhu, D.; Wang, H.; et al. Irisin improves fatty acid oxidation and glucose utilization in type 2 diabetes by regulating the AMPK signaling pathway. Int J Obes (Lond) 2016, 40, 443-451, https://doi.org/10.1038/ijo.2015.199.

51. Naohiro, Y.; Ling, Z.; Dennis, W.; Patrycja, M. D.; Lei, W.; Shougang, Z.; Ping, Z. Irisin counteracts high glucose and fatty acid-induced cytotoxicity by preserving the AMPK-insulin receptor signaling axis in $\mathrm{C} 2 \mathrm{C} 12$ myoblasts. Endocrinology and metabolism 2020, https://doi.org/10.1152/ajpendo.00219.2019.

53. Shim, Y.S.; Kang, M.J.; Yang, S. and Hwang, I. Irisin is a biomarker for metabolic syndrome in prepubertal children. Endocrine journal 2018, 65 (1), 23-31, https://doi.org/10.1507/endocrj.EJ17-0260.

54. Ghoshal, S.; Stevens, J.; Billon, C.; Girardet, C.; Sitaula, S.; Leon, A.; et al. Adropin: An endocrine link between the biological clock and cholesterol homeostasis. Molecular Metabolism 2018, 8, 51- 64, https://doi.org/10.1016/j.molmet.2017.12.002.

55. Su, G.; Sarbani, G.; Liyan Z.; Joseph, R S.; Kyle, S.; McCommis, B. N.; Finck, Gary, D. L. and Andrew A. B. The peptide hormone adropin regulates signal transduction pathways controlling hepatic glucose metabolism in a mouse model of diet-induced obesity. Journal of Biological Chemistry 2019, 294, (36). https://doi.org/10.1074/jbc.RA119.008967.

56. Butler, A.; Tam, C.; Stanhope, K.; Wolfe, B.; Ali, M.; O'Keeffe, M.; St-Onge, M.; Ravussin, E.; Havel, P. Low Circulating Adropin Concentrations with Obesity and Aging Correlate with Risk Factors for Metabolic Disease and Increase after Gastric Bypass Surgery in Humans. The Journal of Clinical Endocrinology \& Metabolism 2012, 97(10), 1, https://doi.org/10.1074/jbc.RA119.008967.

57. Darko, B.; Dinko, M.; Piero, Marin, Z.; Daria, T.; Hadjina T. I. et al. Serum adropin levels are reduced in patients with inflammatory bowel diseases. Scientific Reports. 2020, 10, 9264, https://doi.org/10.1038/s41598-020-66254-9. 